\title{
NOTA
}

\section{USO DE OPALAS BIOGÊNICAS NA IDENTIFICAÇÃO DE SEDIMENTOS LACUSTRES E MARINHOS ${ }^{(1)}$}

\author{
João Luiz Lani ${ }^{(2)}$, Larry Schwandes ${ }^{(3)}$, Sérvulo Batista de \\ Rezende $^{(4)}$, José Antonio Lani ${ }^{(5)}$, Eufran Ferreira do Amaral ${ }^{(6)}$, \\ Alexson de Mello Cunha ${ }^{(7)} \&$ Liovando Marciano da Costa ${ }^{(8)}$
}

RESUMO

\begin{abstract}
Procurou-se utilizar opalas biogênicas, também conhecidas como espículas ou "pó-de-mico", como identificadoras de sedimentos lacustres ou marinhos. Para isso, analisaram-se camadas e horizontes de um Gleissolo Tiomórfico Húmico na região do rio Riacho, Aracruz, ES. Cada amostra de solo foi submetida a tratamentos específicos para o isolamento das espículas; posteriormente, elas foram identificadas em microscópico eletrônico de varredura. Verificou-se a presença de espículas de origem tanto lacustre como marinha e que estas, além de serem úteis na identificação da origem dos sedimentos, podem propiciar, pela sua forma, as condições geoquímicas do ambiente e a distância de transporte dos sedimentos.
\end{abstract}

Termos de indexação: indicadores ambientais, solos hidromórficos, Gleissolos.

\section{SUMMARY: BIOGENIC OPALS IN THE IDENTIFICATION OF LACUSTRINE AND MARINE SEDIMENTS}

This study aimed to identify lacustrine or marine sediments based on biogenic opals (also known as spicules). The layers and horizons of a Humic Gley Thiomorphic soil in the

\footnotetext{
(1) Parte da Tese de Doutorado do primeiro autor apresentada ao Departamento de Solos da Universidade Federal de Viçosa. Recebido para publicação em abril de 2004 e aprovado em abril de 2007.

(2) Professor do Departamento de Solos, Universidade Federal de Viçosa - UFV. Av. PH Rolfs s/n, CEP 36570-000 Viçosa (MG). E-mail: lani@ufv.br

(3) Pesquisador do Soil Department, University of Florida, Gainesville, Florida, USA. E-mail: larryschwandes@yahoo.com

(4) Professor Aposentado do Departamento de Solos, UFV. E-mail: neput@ufv.br

(5) Pesquisador do INCAPER. Estação Experimental do INCAPER, CEP 29900-000 Linhares (ES). E-mail: jalani@incaper.es.gov.br

(6) Pesquisador da Embrapa - ACRE. Rod. BR 364, Km 14, Caixa Postal 321, CEP 69908-970 Rio Branco (AC). E-mail: eufran@buynet.com.br

(7) Técnico do INCRA. AANE 40, Alameda 1, Q1 08, Lote 1, CEP 77006-336 Palmas (TO). E-mail: alexson.cunha@pmj.incra.gov.br

(8) Professor do Departamento de Solos, UFV. E-mail: liovandomc@yahoo.com.br
} 


\begin{abstract}
Riacho catchment area, Aracruz, state of Espirito Santo, Brazil, were analyzed. Each soil sample was specifically treated to isolate the spicules, which were then identified by an electronic microscope. It was possible to verify the presence of lacustrine as well as marine spicules. Moreover, the shape of the opals provided information on the conditions of the geochemical environment and transport distance of the sediments.
\end{abstract}

Index terms: environmental indicators, hydromorphic soils, Gleysoils.

\section{INTRODUÇÃO}

Na tentativa de descobrir novas ferramentas que facilitem a identificação de sedimentos, se lacustres ou marinhos, bem como se estes foram submetidos à movimentação (transporte) por longas distâncias, procurou-se identificar, em amostras de um perfil de Gleissolo Tiomórfico Húmico (Embrapa, 1998; Lani, 1998), a presença de espículas de esponjas, conhecidas também como "pó-de-mico" (Stone et al., 1970; Costa et al., 1992; Schwandes \& Collins, 1994).

Espículas são corpos pontiagudos, como se fossem pequeníssimas agulhas. São partes das esponjas animais aquáticos, multicelulares, de ocorrência em ambiente marinho ou lacustre, considerados um dos mais primitivos do mundo. Concentram e precipitam Si na forma de sílica hidratada (opalas) ou Ca, como carbonato de cálcio (Garrone et al., 1981). Após a morte do animal (esponja), as espículas são incorporadas ao solo ou até às rochas sedimentares. Sua preservação depende da sua constituição química e das condições ambientais onde se depositam.

Esses corpos são conhecidos como "pó-de-mico" devido à coceira que causam quando penetram na pele humana. Podem causar forte reação alérgica em pessoas mais sensíveis. Se esse pó (espículas) for aspirado e adentrar os pulmões, pode causar sérios problemas de saúde. São encontradas em abundância nas regiões de Sete Lagoas, João Pinheiro, Romaria e Lagoa da Prata (Minas Gerais) e em Goianésia (Goiás). Segundo Costa et al. (1992), em todos esses locais, o "pó-de-mico" aparece em condições de drenagem impedida, com evidências de hidromorfismo e associado aos Gleissolos e Organossolos. Em Minas Gerais, são misturadas ao barro (solo) na fabricação de tijolos refratários, utilizados em fornos de alta temperatura.

Assim, este trabalho teve por objetivos descobrir novas ferramentas que facilitem a identificação de sedimentos lacustres ou marinhos e identificar a presença de espículas em um Gleissolo Tiomórfico Húmico.

\section{MATERIAL E MÉTODOS}

A fim de atingir os objetivos propostos, coletaramse amostras de um perfil de Gleissolo Tiomórfico húmico, localizado na Fazenda AGRIL, a $5 \mathrm{~km}$ da localidade de Barra do Riacho, em direção a Regência (foz do rio Doce), do lado esquerdo, no município de Aracruz, Espírito Santo (Quadros 1 e 2).

No procedimento de descrição do perfil adotou-se o método recomendado por Lemos \& Santos (1984). Tanto as análises físicas (densidade do solo, equivalente de umidade e condutividade elétrica) quanto as químicas (complexo sortivo, $\mathrm{N}, \mathrm{P}$ disponível e ataque sulfúrico - $\mathrm{SiO}_{2}, \mathrm{Al}_{2} \mathrm{O}_{3}, \mathrm{Fe}_{2} \mathrm{O}_{3}$ e $\mathrm{TiO}_{2}$ ) foram realizadas conforme métodos descritos e aplicados rotineiramente em laboratórios da UFV (Vettori, 1969; Embrapa, 1979; Defellipo \& Ribeiro, 1981). O P remanescente foi determinado segundo Alvarez V. et al. (1993).

A determinação do C orgânico foi feita pelo método de Walkley-Black (Jackson, 1979); o S total foi obtido por ataque com $\mathrm{HCl}$ 1:1, precipitação gravimétrica com $\mathrm{BaCl}_{2}$ e determinação gravimétrica. $\mathrm{O} \mathrm{pH}$ foi determinado em água e em $\mathrm{KCl}\left(1 \mathrm{~mol} \mathrm{~L}^{-1}\right)$, na relação $1: 2,5$. O S, pH e condutividade elétrica foram determinados segundo procedimentos recomendados por Embrapa (1979).

Após a coleta do material de solo (amostras), este foi seco e passado em peneira de $2 \mathrm{~mm}$. No tratamento inicial das amostras, a matéria orgânica (MO) foi eliminada com $\mathrm{H}_{2} \mathrm{O}_{2} 30$ volumes. Considerou-se a eliminação da matéria orgânica com o término da efervescência, pelo uso da $\mathrm{H}_{2} \mathrm{O}_{2}$, e a apresentação da cor mais clara do material de solo. Posteriormente, a argila foi dispersa com hexametafosfato de sódio (calcon) e eliminada. Separou-se a fração silte da areia por peneiramento. Finalmente, com uso do bromato de zinco (líquido de alta densidade), separaram-se as espículas da fração silte restante. Os procedimentos mais detalhados utilizados no isolamento das opalas biogênicas e sua identificação são descritos a seguir: após a pesagem de $10 \mathrm{~g}$ de solo, este foi colocado em béquer de $600 \mathrm{~mL}$; posteriormente, adicionaram-se cerca de $300 \mathrm{~mL}$ de água deionizada e $25 \mathrm{~mL}$ de $\mathrm{H}_{2} \mathrm{O}_{2}$ 30 volumes. Em seguida, o béquer foi colocado sobre chapa quente, à temperatura de $60{ }^{\circ} \mathrm{C}$, aproximadamente por $24 \mathrm{~h}$. Adicionou-se $\mathrm{H}_{2} \mathrm{O}_{2} 30$ volumes até que a solução não apresentasse efervescência e a cor do material de solo fosse mais clara. Com este procedimento, considerou-se eliminada a MO; depois disso, adicionaram-se ao béquer $50 \mathrm{~g} \mathrm{~L}^{-1} \mathrm{de}$ hexametafosfato de sódio a $50 \mathrm{~g} \mathrm{~L}^{-1}$, com o objetivo de dispersar a argila e eliminá-la (Embrapa, 1979). A seguir, separou-se a fração areia do silte, utilizando- 
Quadro 1. Principais características morfológicas do Gleissolo Tiomórfico húmico e dos biogênicos identificados

Horizonte/camada Profundidade Característica morfológica $\frac{\text { Presença de espícula }}{\text { Lacustre Marinha }}$ Observação

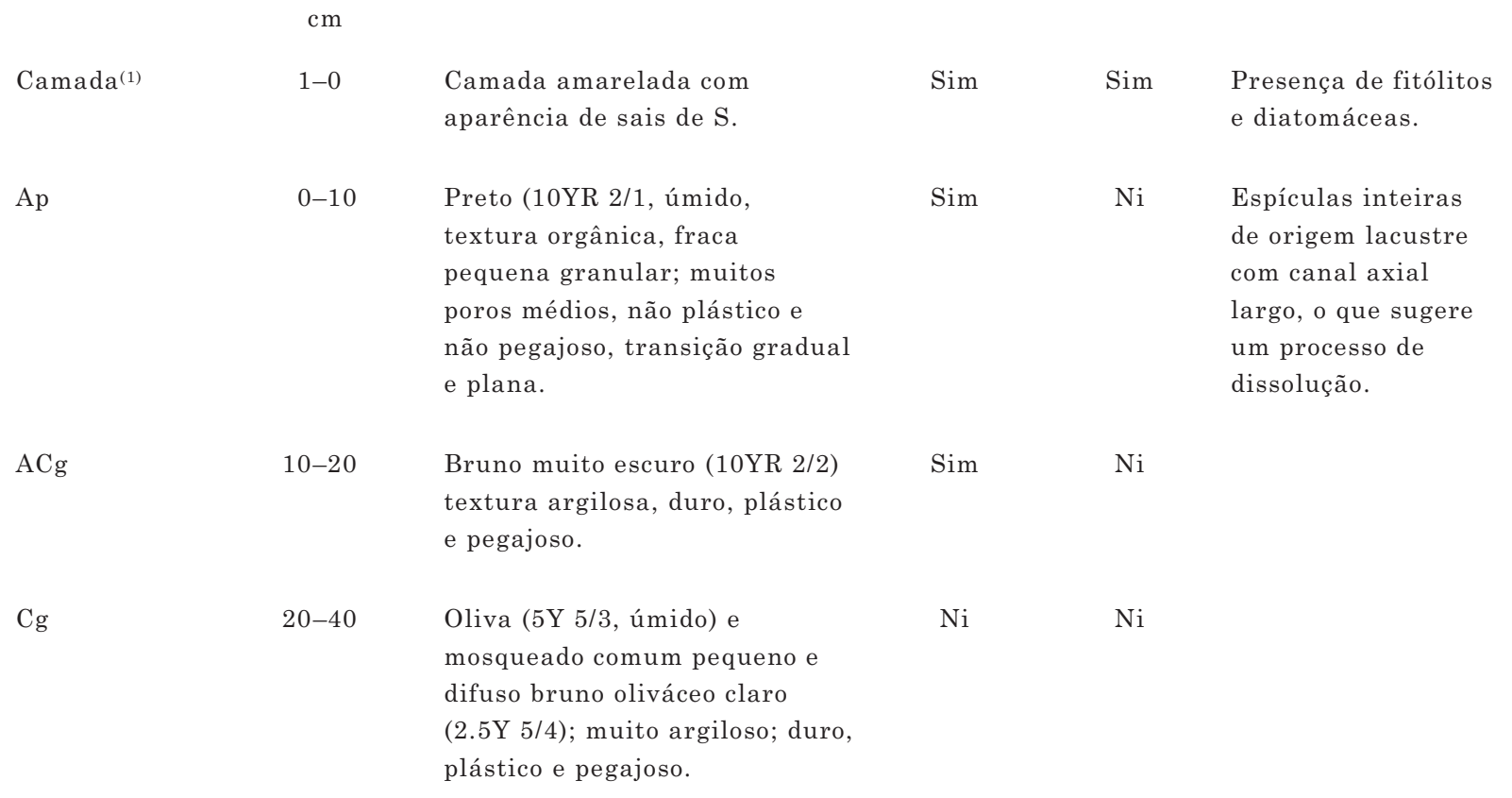

(1) Preferiu-se denominar "camada” em vez de horizonte, em razão de ser uma crosta salina. Ni: não-identificado.

Quadro 2. Características físicas e químicas do Gleissolo Tiomórfico húmico

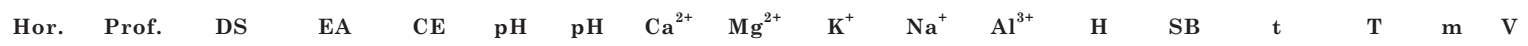
$\mathrm{H}_{2} \mathrm{O} \quad \mathrm{KCl}$

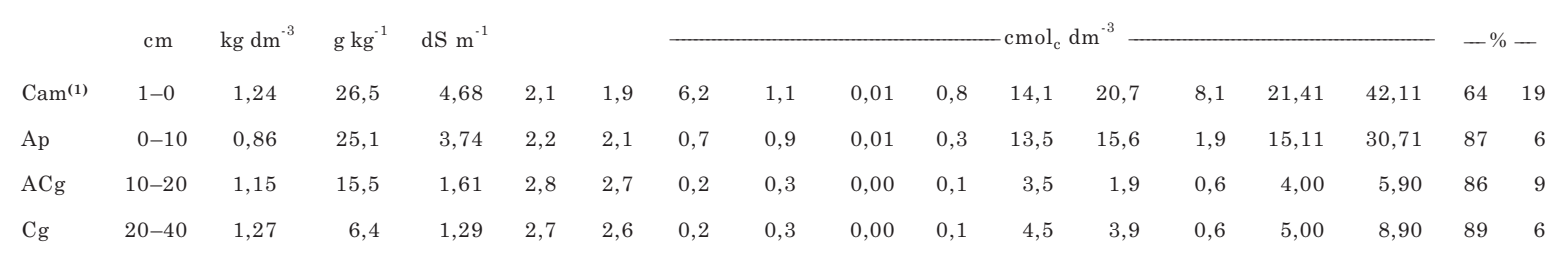

\begin{tabular}{|c|c|c|c|c|c|c|c|c|c|c|c|c|c|}
\hline & & C & $\mathrm{N}$ & $\mathrm{C} / \mathrm{N}$ & $\mathbf{P}$ & Prem & $\mathrm{S}$ & $\mathrm{SiO}_{2}$ & $\mathrm{Al}_{2} \mathrm{O}_{3}$ & $\mathrm{Fe}_{2} \mathrm{O}$ & $\mathrm{TiO}_{2}$ & $\mathbf{K i}$ & $\mathbf{K r}$ \\
\hline & $\mathrm{cm}$ & - & ${ }^{-3}$ & & $\mathrm{mg} \mathrm{dm}{ }^{-3}$ & $\mathrm{mg} \mathrm{L}^{-1}$ & 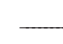 & 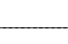 & $\mathrm{g} \mathrm{dm}^{-3}$ & 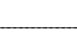 & 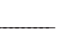 & & \\
\hline $\mathrm{Cam}^{(1)}$ & $1-0$ & 171 & 0,54 & 31,71 & 0,8 & 14,0 & 49,5 & 67 & 22 & 154 & 4 & - & - \\
\hline Ap & $0-10$ & 111 & 1,44 & 7,7 & 4,6 & 12,3 & 7,9 & 66 & 20 & 23 & 3 & - & - \\
\hline Acg & $10-20$ & 8 & 0,1 & 8,0 & 0,5 & 18,6 & 6,2 & 69 & 55 & 21 & 5 & 2,13 & 1,71 \\
\hline $\mathrm{Cg}$ & $20-40$ & 9 & 0,08 & 11,3 & 0,2 & 25,3 & 3,3 & 33 & 26 & 9 & 2 & 2,16 & 1,76 \\
\hline
\end{tabular}

(1) Preferiu-se denominar "camada" em vez de horizonte, em razão de ser uma crosta salina. DS: densidade do solo; EA: equivalente de umidade; CE: condutividade elétrica. 
se uma peneira de $20 \mathrm{~cm}$ de diâmetro e malha de 0,053 mm ( $\left.\mathrm{n}^{\circ} 270\right)$. A ação seguinte foi proceder à separação das partículas biogênicas da fração silte do solo. Neste caso, levou-se o béquer à estufa a $100{ }^{\circ} \mathrm{C}$, até evaporar toda a água. Posteriormente, foram transferidos para um tubo de centrífuga cerca de $250 \mathrm{mg}$ de fração silte e adicionaram-se $5 \mathrm{~mL}$ de solução de $144 \mathrm{~g}$ de bromato de zinco em $50 \mathrm{~mL}$ de água, densidade de $2,3 \mathrm{~g} \mathrm{~cm}^{-3}$. Centrifugou-se o material a $2.000 \mathrm{rpm}$ por $10 \mathrm{~min}$. Com isso, o material de solo permaneceu no fundo do tubo da centrífuga e as partículas biogênicas, no sobrenadante. Coletouse o sobrenadante e adicionou-se água o suficiente para cobrir a amostra, repetindo a centrifugação na mesma rotação e tempo, para lavagem da amostra. Em seguida, descartou-se a água e adicionaram-se ao tubo de centrifuga $2 \mathrm{~mL}$ de água; agitou-se e retiraram-se $20 \mu \mathrm{L}$ da solução, a qual foi distribuída uniformemente em uma lâmina de microscópio; com o uso de microscópio de varredura da University of Florida, procedeu-se à identificação e caracterização das opalas biogênicas.

\section{RESULTADOS E DISCUSSÃO}

Verificou-se na camada superficial (crosta salina) do Gleissolo Tiomórfico húmico (Camada 1) a presen- ça de espículas de origem tanto lacustre como marinha, além de fitólitos e diatomáceas (Quadro 1 e Figura 1). Nos horizontes subsuperficiais a essa camada (Ap e ACg), foram identificadas somente espículas lacustres, além de fitólitos e diatomáceas (Figura 2). No horizonte $\mathrm{Cg}(20-40 \mathrm{~cm})$ não foi identificado qualquer tipo de espícula. Supõe-se que a presença de espículas lacustres no perfil se deve ao fato de o local onde foram coletadas as amostras de solo ter sido um antigo leito de rio, conforme informação de um morador da região. Atualmente, a área está sendo invadida pelo mar (maré). Neste caso, o tiomorfismo está sendo formado de cima para baixo, por influência da maré. Essa explicação é corroborada pela presença de elevados teores de $\mathrm{S}$ e maiores valores da condutividade elétrica na superfície, que decrescem com a profundidade (Quadro 2). Assim, sugere-se que as espículas estão identificando a origem dos sedimentos, isto é, na parte superior do perfil existem sedimentos tanto marinhos quanto lacustres (mistura) e, na parte de baixo, somente lacustre, em razão de o local ser um antigo leito de rio.

As espículas podem também contribuir com a identificação dos sedimentos quanto à distância do transporte. Espículas inteiras, sem estarem quebradas, como no horizonte Ap (Figura 2), indicam que os sedimentos foram transportados a curta distância, pois elas apresentam certa fragilidade a

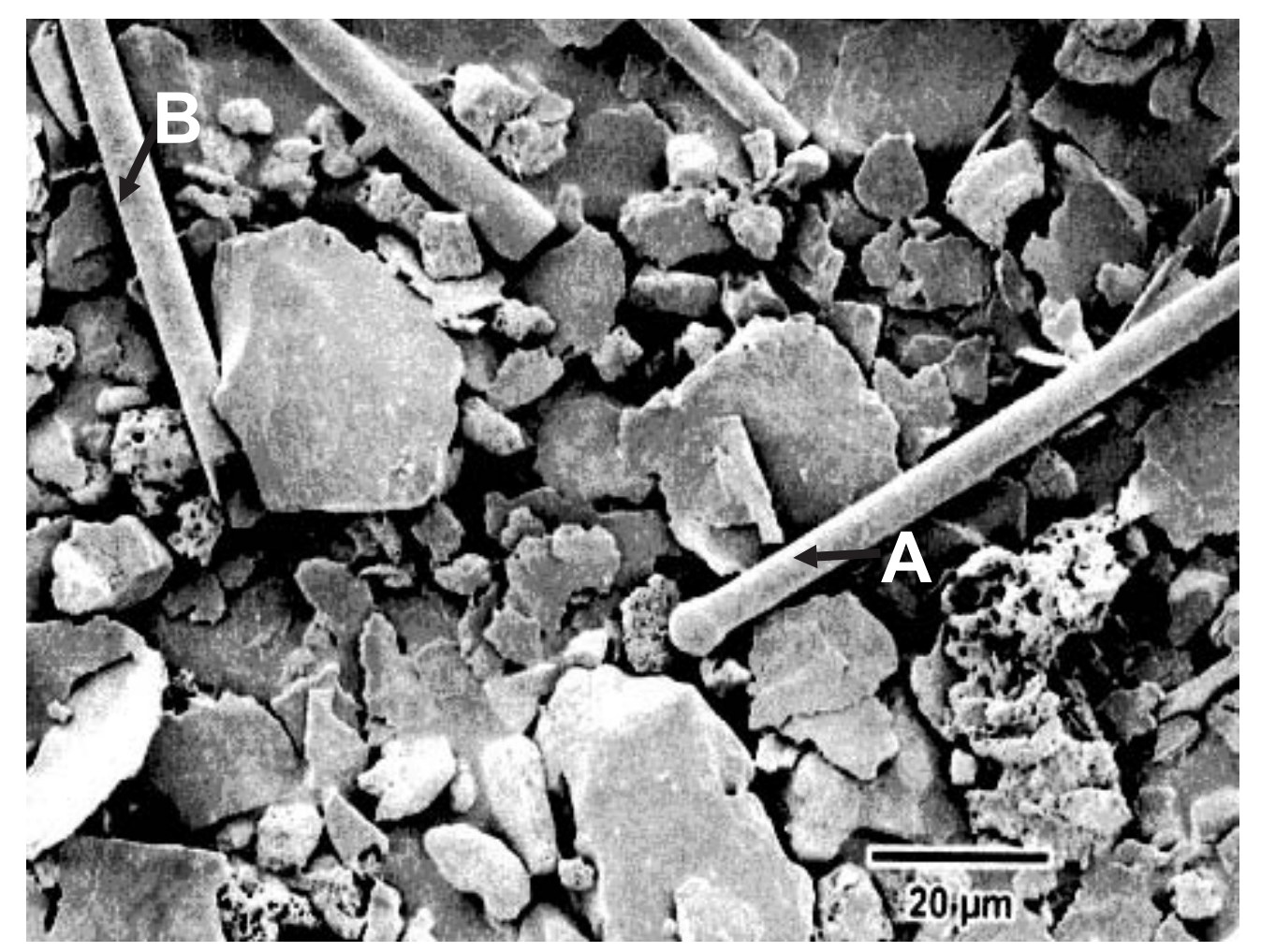

Figura 1. Opalas biogênicas identificadas na camada 1. A - espícula de ambiente marinho (cotonete). B espícula de ambiente lacustre (formas tubulares). Foto de microscopia eletrônica de varredura University of Florida. 


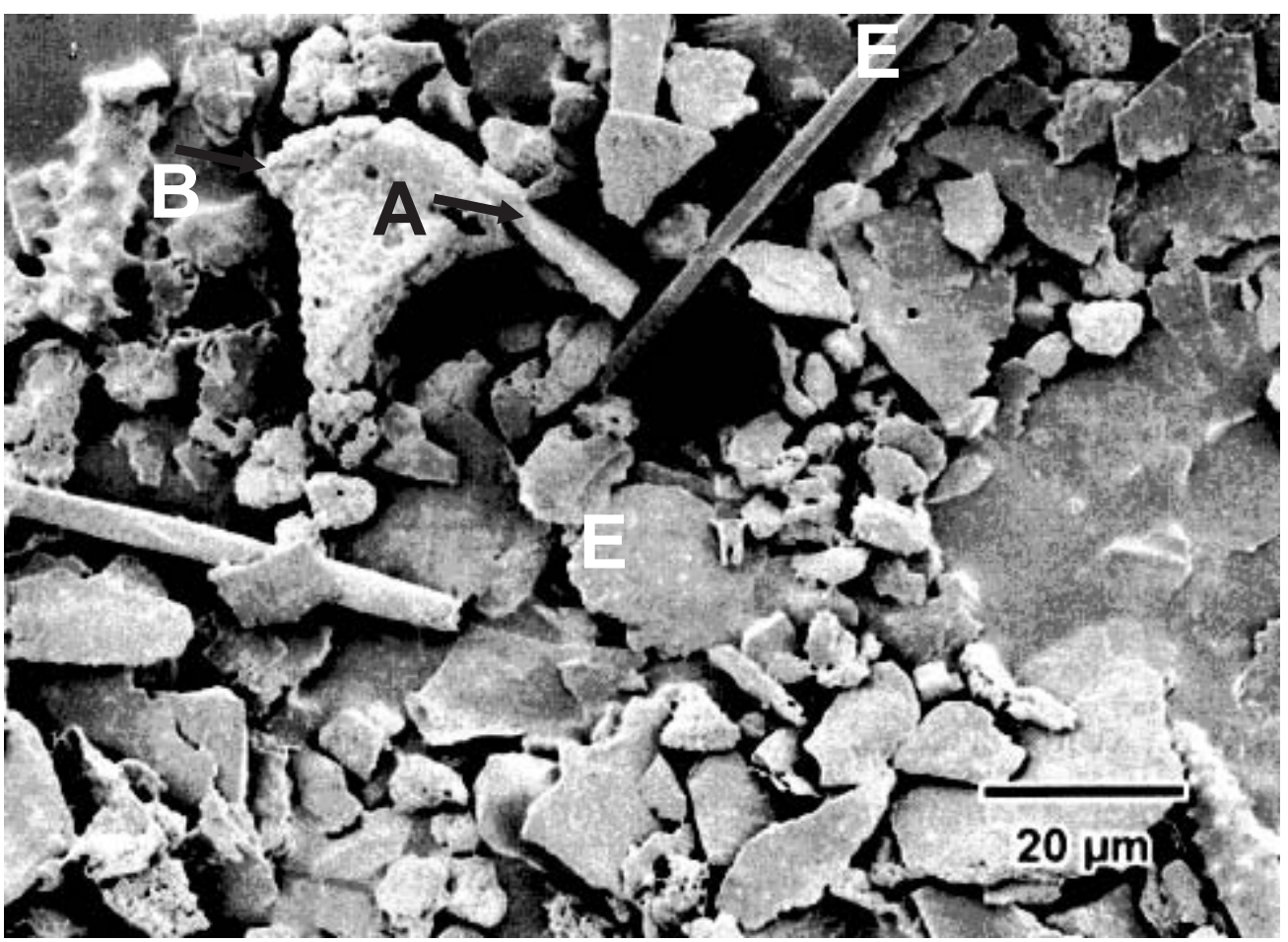

Figura 2. Opalas biogênicas identificadas no horizonte Ap. A - fitólitos de gramíneas; B - espículas de esponjas de ambiente lacustre. Foto de microscopica eletrônica de varredura - University of Florida.

embates físicos (Smithson, 1959; Jones \& Beavers; 1963; Hart \& Fuller, 1974).

\section{CONSIDERAÇOES FINAIS}

As espículas podem auxiliar na identificação de sedimentos (solo) quanto à sua origem: marinha ou lacustre. $\mathrm{O}$ comportamento delas pode também auxiliar na compreensão das condições geoambientais; pela sua condição física, é possível inferir se o sedimento foi transportado por longas distâncias (embates físicos).

\section{LITERATURA CITADA}

ALVAREZ, V., V.H.; DIAS, L.E. \& OLIVEIRA, J.A. Determinação do fósforo remanescente. Viçosa, MG, Universidade Federal de Viçosa, 1993. 11p.

COSTA, L.M.; KELLER, W.D. \& JOHNS, W.D. Espículas de esponjas em solos de João Pinheiro, Minas Gerais. R. Ceres, 39:597-603, 1992.

DEFELIPO, B.V. \& RIBEIRO, A.C. Análise química do solo. Viçosa, MG, Universidade Federal de Viçosa, 1981. 12p. (Boletim de Extensão, 1981)
EMPRESA BRASILEIRA DE PESQUISA AGROPECUÁRIA EMBRAPA. Centro Nacional de Pesquisa de Solos. Serviço Nacional de Levantamento e Conservação de Solos. Manual de métodos de análise de solo. Rio de Janeiro,1979. Não paginado.

EMPRESA BRASILEIRA DE PESQUISA AGROPECUÁRIA . EMBRAPA.Centro Nacional de Pesquisa de Solos. Sistema brasileiro de classificação de solos. Rio de Janeiro, 1998. 412p.

GARRONE, R.; SIMPSON, T.L. \& POTTU-BOUMENDIL, J. Ultrastructure and deposition of silica in sponges. In: SIMPSON, T.L. \& VOLCANI, B.E, eds. Silicon and siliceous structure in biological systems. New York, Springer-Verlag, 1981. p.495-525.

HART, C.W. \& FULLER, S.L.H. Pollution ecology of freshwater invertebrates. New York, Academy Press, 1974.389p.

JACKSON, M.L. Soil chemical analysis - Advanced course. 2.ed. Madison, University of Wisconsin, 1979. 895p.

JONES, R.L. \& BEAVERS, A.H. Sponges spicules in lllinois soils. Soil Sci. Soc. Am. Proc., 27:438-440, 1963.

LANI, J.L. Deltas dos rios Doce e Itapemirim: Solos, com ênfase nos tiomórficos, água e impacto ambiental do uso. Viçosa, MG, Universidade Federal de Federal de Viçosa, 1998. 169p. (Tese de Doutorado) 
LEMOS, R.C. \& SANTOS, D.R. Manual de descrição e coleta de solo no campo. Campinas, Sociedade Brasileira de Ciência do Solo/ Comissão de Método de Trabalho de Campo/Serviço Nacional de Levantamento e Conservação de Solos, 1984. 46p.

SCHWANDES, L.P. \& COLLINS, M.E. Distribution and significance of freshwater sponge spicules in selected Florida soils. Trans. Am. Microsc. Soc., 113:242-257, 1994.
SMTHSON, F. Opal sponge spicules in soils. J. Soil Sci., 10:105109, 1959.

STONE, E.L.; DAWSON, J.E. \& ZEEUW, C.D. Dermatite caused by sponge spicules. Soil Sci. Soc. Am. Proc., 34:151$152,1970$.

VETTORI, L. Métodos de análise de solos. Rio de Janeiro, Equipe de Pedologia e Fertilidade do Solo, 1969. 34p. (Boletim Técnico, 7) 